\title{
An immersion of a square in 4-edge-connected graphs
}

\author{
Ken-ichi KAWARABAYASHI ${ }^{1}$ and Yusuke KOBAYASHI ${ }^{2}$ \\ ${ }^{1}$ National Institute of Informatics \\ ${ }^{2}$ University of Tokyo
}

\begin{abstract}
For an undirected graph $G$ and its four distinct vertices $v_{1}, v_{2}, v_{3}, v_{4}$, an immersion of $\left(v_{1}, v_{2}, v_{3}, v_{4}\right)$ is a subgraph of $G$ that consists of four edge-disjoint paths $P_{1}, P_{2}, P_{3}, P_{4}$ such that $P_{i}$ connects $v_{i}$ and $v_{i+1}$ for $i=1,2,3,4$, where $v_{5}=v_{1}$. We show that every 4-edgeconnected graph $G=(V, E)$ has an immersion of $\left(v_{1}, v_{2}, v_{3}, v_{4}\right)$ for any $v_{1}, v_{2}, v_{3}, v_{4} \in V$, and it can be found in linear time.
\end{abstract}

\section{KEYWORDS}

graph algorithm, immersion, edge-connectivity, edge-disjoint paths

Characterizing an undirected graph $G=(V, E)$ without a cycle through $v_{1}, v_{2}, v_{3}, v_{4}$ (for four distinct vertices $\left.v_{1}, v_{2}, v_{3}, v_{4} \in V\right)$ in this order is a very hard problem. Indeed, in a series of paper [6]-[8], Yu has characterized a graph without a path through $v_{1}, v_{2}, v_{3}, v_{4}$ in this order, but this weaker form is already a deep and very hard theorem. At the moment, it is without reach to find such a characterization for a graph without a cycle through $v_{1}, v_{2}, v_{3}, v_{4}$ in this order. If we impose some connectivity condition, this may be feasible. In fact it follows from Thomas and Wollan's result [5] (saying that every $10 k$-connected graph is $k$-linked) that such a cycle exists if a given graph is 40 -connected. But on the other hand, the connectivity is far from best possible.

In this paper, we seek for the edge-disjoint version of this problem. For an undirected graph $G=(V, E)$ and for four distinct vertices $v_{1}, v_{2}, v_{3}, v_{4} \in V$, an immersion of $\left(v_{1}, v_{2}, v_{3}, v_{4}\right)$ is a subgraph of $G$ that consists of four edge-disjoint paths $P_{1}, P_{2}, P_{3}, P_{4}$ such that $P_{i}$ connects $v_{i}$ and $v_{i+1}$ for $i=1,2,3,4$, where $v_{5}=v_{1}$. Note that if we replace "edge-disjoint" by "vertex-disjoint", then the union of the paths $P_{1}, P_{2}, P_{3}, P_{4}$ gives rise to a cycle through $v_{1}, v_{2}, v_{3}, v_{4}$ in this order. Thus we may think of our problem as a counterpart to the above problem in view of edge-disjoint paths. Let us observe that the concept "immersion" is an important concept in graph theory, which is well-studied in [4].

We show that every 4-edge-connected graph $G=$

Received November 14, 2011; Accepted December 22, 2011

1)k_keniti@nii.ac.jp, ${ }^{2)}$ kobayashi@mist.i.u-tokyo.ac.jp

DOI: 10.2201/NiiPi.2012.9.7
$(V, E)$ has an immersion of $\left(v_{1}, v_{2}, v_{3}, v_{4}\right)$ for any $v_{1}, v_{2}, v_{3}, v_{4} \in V$, and it can be found in linear time.

Theorem 1. For any 4-edge-connected graph $G=$ $(V, E)$ and for any distinct vertices $v_{1}, v_{2}, v_{3}, v_{4} \in V$, there exists an immersion of $\left(v_{1}, v_{2}, v_{3}, v_{4}\right)$ in G. Furthermore, it can be found in $O(|E|)$ time.

Let us point out that if we use Huck's result [1] (saying that every $(k+2)$-edge-connected graph is weakly $k$-linked for any even $k$ ), the edge-connectivity in Theorem 1 is at most 6 . Thus our edge-connectivity is better in this sense. Indeed, our edge-connectivity is best possible in a sense (see later).

Before giving a proof of this theorem, we give some remarks. When the graph has maximum degree at most three, "edge-disjoint" and "vertex-disjoint" clearly mean the same condition. This implies that, in such graphs, the problem of finding an immersion of $\left(v_{1}, v_{2}, v_{3}, v_{4}\right)$ is equivalent to the problem of finding a cycle through $\left(v_{1}, v_{2}, v_{3}, v_{4}\right)$ in this order, which is known to be a very difficult problem, as mentioned, even for cubic graphs (as given in [6]-[8], there are many cubic graphs that do not have a cycle through $\left(v_{1}, v_{2}, v_{3}, v_{4}\right)$ in this order). Therefore, when we remove the assumption of 4-edge-connectivity, we know no polynomial-time algorithm for this problem without using Robertson and Seymour's algorithm [3] (and a faster time complexity algorithm in [2]) based on the graph minor theory. It is natural to ask at this point why we do not consider the weaker condition that the minimum degree being at least four, but in fact this 
weaker restriction would not gain us anything. Suppose that we are given an arbitrary graph $G=(V, E)$ that may have degree three vertices, and four distinct vertices $v_{1}, v_{2}, v_{3}, v_{4} \in V$. Then attach by two edges to each vertex in $G$ a constant-sized graph of high minimum degree. The resulting graph has minimum degree high, but clearly this modification does not affect the existence of an immersion of $\left(v_{1}, v_{2}, v_{3}, v_{4}\right)$. This example shows that 4 -edge-connectivity is necessary. Thus we really need to stick the 4-edge-connectivity in our proof.

Proof of Theorem 1. Since $G$ is 4-edge-connected, there exist four edge-disjoint paths $Q_{1}, Q_{2}, Q_{3}$, and $Q_{4}$ such that $Q_{1}$ and $Q_{2}$ are $v_{1}-v_{2}$ paths, and $Q_{3}$ and $Q_{4}$ are $v_{1}-v_{4}$ paths. If $v_{3}$ is contained in $Q_{i}$ for some $i \in\{1,2,3,4\}$, then we obtain an immersion of $\left(v_{1}, v_{2}, v_{3}, v_{4}\right)$ by concatenating $Q_{1}, Q_{2}, Q_{3}$, and $Q_{4}$ in an appropriate order.

Otherwise, we take four edge-disjoint paths $R_{1}, R_{2}, R_{3}, R_{4}$ from $v_{3}$ to $Q_{1} \cup Q_{2} \cup Q_{3} \cup Q_{4}$ such that $V\left(R_{j}\right) \cap V\left(Q_{1} \cup Q_{2} \cup Q_{3} \cup Q_{4}\right)=\left\{u_{j}\right\}$ for $j=1,2,3,4$ (possibly $u_{j}=u_{k}$ for $j \neq k$ ). If $\left|\left\{j \mid u_{j} \in V\left(Q_{i}\right)\right\}\right| \geq 2$ for some $i \in\{1,2,3,4\}$, then we obtain an immersion of $\left(v_{1}, v_{2}, v_{3}, v_{4}\right)$ in the same way as the case of $v_{3} \in V\left(Q_{i}\right)$. Otherwise, we may assume that $u_{i} \in V\left(Q_{i}\right)$ for $i=1,2,3,4$. In this case, we obtain an immersion of $\left(v_{1}, v_{2}, v_{3}, v_{4}\right)$ by concatenating $Q_{1}, Q_{2}\left[v_{2}, u_{2}\right], R_{2}, R_{3}, Q_{3}\left[u_{3}, v_{4}\right], Q_{4}$ in this order, where $Q_{2}\left[v_{2}, u_{2}\right]$ denotes the subpath of $Q_{2}$ between $v_{2}$ and $u_{2}$, and $Q_{3}\left[u_{3}, v_{4}\right]$ is defined in a similar way.

Obviously, the above procedures can be done in $O(|E|)$ time by a flow algorithm, which completes the proof.

\section{Acknowledgement}

Supported by Grant-in-Aid for Scientific Research and by the Global COE Program "The research and training center for new development in mathematics", NEXT, Japan.

\section{References}

[1] A. Huck, "A sufficient condition for graphs to be weakly k-linked," Graphs and Combinatorics, vol.7, pp.323351, 1991

[2] K. Kawarabayashi, Y. Kobayashi and B. Reed, "The disjoint paths problem in quadratic time", J. Combin. Theory Ser. $B$, to appear.

[3] N. Robertson and P.D. Seymour, "Graph minors. XIII. The disjoint paths problem," J. Combin. Theory Ser. B, vol.63, pp.65-110, 1995.

[4] N. Robertson and P.D. Seymour, "Graph minors. XXIII. Nash-Williams' immersion conjecture," J. Combin. Theory Ser. B, vol.100, pp.181-205, 2010.
[5] R. Thomas and P. Wollan, "An improved linear edge bound for graph linkages," Eur. J. Comb., vol.26, pp.309324, 2005.

[6] X. Yu, "Disjoint paths in graphs I, 3-planar graphs and basic obstructions," Annals of Combinatorics, vol.7, pp.89-103, 2003.

[7] X. Yu, "Disjoint paths in graphs II, a special case," Annals of Combinatorics, vol.7, pp.105-126, 2003.

[8] X. Yu, "Disjoint paths in graphs III, characterization," Annals of Combinatorics, vol.7, pp.229-246, 2003.

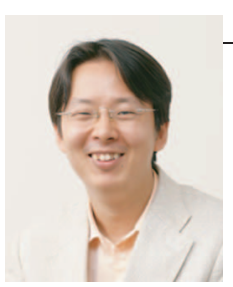

\section{Ken-ichi KAWARABAYASHI}

Ken-ichi KAWARABAYASHI is Professor of National Institute of Informatics. He received Bs, MA, Ph.D from Keio University in 1998, 2000, 2001, respectively. $\mathrm{He}$ is an editor of several international well-known journals, including Siam J. Discrete Mathematics and J. Graph Theory. He has won several domestic prizes, including IBM Science Prize in Computer Science in 2008.

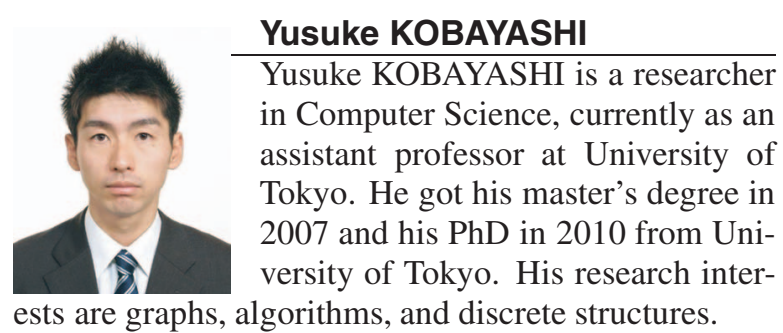

Yusuke KOBAYASHI is a researcher in Computer Science, currently as an assistant professor at University of Tokyo. He got his master's degree in versity of Tokyo. His research interests are graphs, algorithms, and discrete structures. 